\title{
Limbic system-associated membrane protein as a potential target for neuropsychiatric disorders
}

\author{
Jürgen Innos ${ }^{1}$, Kati Koido ${ }^{1}$, Mari-Anne Philips ${ }^{1}$ and Eero Vasar ${ }^{1,2}$ * \\ ' Department of Physiology, University of Tartu, Tartu, Estonia \\ ${ }^{2}$ Estonian Academy of Sciences, Tallinn, Estonia
}

Edited by:

Michel Bourin, University of Nantes,

France

Reviewed by:

Michel Bourin, University of Nantes, France

Martine Hascoet, University of

Nantes, France

\section{${ }^{*}$ Correspondence:}

Eero Vasar, Department of Physiology, University of Tartu, Biomedicum, 19 Ravila Street, 50411 Tartu, Estonia. e-mail:eero.vasar@ut.ee
The studies performed in laboratory animals and psychiatric patients suggest a possible role of limbic system-associated membrane protein (LAMP) in the mechanisms of psychiatric disorders. Stressful manipulations and genetic invalidation have revealed a role of the Lsamp gene in the regulation of anxiety in rodents. Besides that, Lsamp-deficient mice display reduced aggressiveness and impaired adaptation in novel and stressful environments. The behavioral effects of amphetamine were blunted in genetically modified mice. Recent pharmacological and biochemical studies point toward altered function of GABA-, 5-hydroxytryptamine-, and dopaminergic systems in Lsamp-deficient mice. Moreover, we found an association between the gene polymorphisms of LSAMP and major depressive disorder (MDD). Patients suffering from MDD had significantly increased ratio between risk and protective haplotypes of the LSAMP gene compared to healthy volunteers. However, the impact of these haplotypes for the function of LAMP is not clear and remains to be elucidated in future studies.

Keywords: limbic system-associated membrane protein, dopamine, GABA, 5-hydroxytryptamine, anxiety, genetic polymorphisms, major depressive disorder, panic disorder

\section{INTRODUCTION}

The limbic system-associated membrane protein (LSAMP) gene gives rise to LAMP, which is a 64 - to 68 -kDa heavily glycosylated protein, structurally characterized by three immunoglobulin (Ig) domains (Pimenta et al., 1996). LAMP protein is expressed on the surface of somata and proximal dendrites of neurons (Zacco et al., 1990) where it integrates via glycosyl-phosphatidyl-inositol (GPI) anchor (Pimenta et al., 1996). LAMP protein has been shown to be specific to the cortical and sub-cortical limbic-associated regions (e.g., perirhinal cortex, cingulate cortex, amygdala, hippocampus, and striatum) of the developing and adult brain (Levitt, 1984; Horton and Levitt, 1988; Pimenta etal., 1996; Reinoso etal., 1996). Despite the name, LAMP is not expressed only in the limbic-associated areas, but also less intensely in the midbrain and hindbrain regions (Reinoso et al., 1996). A 99\% amino acid sequence identity between human and rodent LAMP (Pimenta et al., 1996) indicates strong phylogenetic conservation of the protein structure and associated functional properties. Several cell culture experiments suggest that LAMP mediates axon targeting and growth in the brain (Keller et al., 1989; Pimenta et al., 1995; Mann et al., 1998; Gil et al., 2002).

\section{FUNCTIONAL STUDIES DEMONSTRATING A ROLE OF THE} Lsamp GENE IN THE REGULATION OF EMOTIONAL BEHAVIOR The first evidence for a role of the Lsamp gene in the regulation of emotional behavior came from a study where male Wistar rats were selected according to their exploratory behavior in the elevated plus-maze model of anxiety. Animals with lower exploratory activity (increased anxiety) had elevated levels of the Lsamp transcript in the periaqueductal gray (Nelovkov et al., 2003). In the same rats, an increase of Lsamp gene expression was also noticed in the amygdala, but not in the frontal cortex (Nelovkov et al., 2006). Exposure of rats to cat odor, another model of anxiety in rodents, also increased the expression of Lsamp transcript in the amygdala (Kõks et al., 2004). These findings were extended by Alttoa etal. (2010) demonstrating that the transcript for Lsamp was more expressed in the raphe, hippocampus, and frontal cortex of rats displaying reduced exploratory activity in the motility box. Lamprecht et al. (2009) established that fear conditioning caused changes in the Lsamp transcript expression in the amygdala of rats. Altogether, rodent studies indicate that increased level of the Lsamp transcript in several brain areas is related with increased trait anxiety (Nelovkov et al., 2003, 2006; Alttoa et al., 2010), acute fear reaction (Kõks et al., 2004), and fear conditioning (Lamprecht et al., 2009).

\section{EVIDENCE FROM STUDIES WITH Lsamp-DEFICIENT MICE SUPPORTING ITS ROLE IN THE REGULATION OF EMOTIONAL BEHAVIOR}

Two different Lsamp gene knockout mouse lines have been created. The first (Catania et al., 2008) was created by deleting exon 2 and the mice were back-crossed more than 10 generations onto the C57BL/6J background. The second (Innos et al., 2011) was created by our research team by deleting exon $1 \mathrm{~b}$ and we used $\mathrm{F} 2$ hybrids $[(129 \mathrm{~S} 6 / \mathrm{SvEvTac} \times \mathrm{C} 57 \mathrm{BL} / 6) \times(129 \mathrm{~S} 6 / \mathrm{SvEvTac} \times \mathrm{C} 57 \mathrm{BL} / 6)]$ for the behavioral experiments, because the congenic footprint effect does not allow to achieve a pure background by backcrossing and besides, it has been argued that a robust behavioral phenotype, detectable over and above the behavioral variability caused by a mixed genetic background, obviates the need for back-crosses (Schalkwyk et al., 2007). Several cell culture experiments suggest that LAMP mediates axon targeting and growth in the brain (Keller 
et al., 1989; Mann et al., 1998; Gil et al., 2002). However, obvious lack of deviations in the brain organization of Lsamp-deficient mouse line, lacking exon 2 of the Lsamp gene, revealed that LAMP is rather mediating finely specialized aspects of circuit formation and maturation of the limbic system (Catania et al., 2008). These two mouse lines have remarkably similar phenotypes: both display no changes in sensory and motor development, are slightly hyperactive in novel environments, and perform more open arm entries and head-dips, and spend more time on open arms in the elevated plus-maze. However, there are also differences between the phenotypes: the first Lsamp gene knockout mouse line, generated in the Vanderbilt University (U.S.A.), exhibited a pronounced deficit in spatial memory acquisition in the water maze and poorly sustained CA1 long-term potentiation (Qiu et al., 2010). The second Lsamp gene knockout mouse line, generated by us, performed normally in the water maze and also displayed no learning deficiencies in the active avoidance test, another learning paradigm (Innos et al., 2011). We have also seen several unique phenotypic differences in our Lsamp gene knockout mice, such as decreased body weight, lack of whisker trimming, decreased aggressive behavior and reduced swimming speed (Innos et al., 2011). A possible explanation for the reduced agonistic and aggressive behavior could be the increased tone of 5-hydroxytryptamine (5-HT) system in the forebrain structures established in male Lsamp-deficient mice (Innos et al., 2013).

In the pharmacological studies we have found that the anxiolytic effect of diazepam is stronger in Lsamp-deficient mice compared to their wild-type littermates. Moreover, the ratio of $\mathrm{GABA}_{\mathrm{A}}$ receptor subunit genes is shifted in favor of the alpha2 subunit compared to the alphal subunit in the temporal lobe of Lsamp-deficient mice. This could be a possible reason for the reduced anxiety as well as the increased anxiolytic effect of diazepam in Lsamp-deficient mice (Innos et al., 2011). Besides that the motor stimulant effect of amphetamine was significantly weaker in young Lsamp-deficient mice (Innos et al., 2013). Moreover, amphetamine did not cause place preference in these mice. The reduced action of amphetamine was accompanied by reduced expression of dopamine transporter, a major target of amphetamine, gene in the mesencephalon. The reduced behavioral effects were accompanied by increased levels and reduced turnover of 5-HT in the forebrain structures in response to amphetamine administration (Innos et al., 2013). Altogether, our recent pharmacological and biochemical studies point toward changes in the function of GABA-, 5-HT-, and dopaminergic systems in Lsamp-deficient mice.

\section{INFLUENCE OF ENVIRONMENTAL MANIPULATIONS ON THE BEHAVIOR OF Lsamp-DEFICIENT MICE}

In order to study the impact of environmental manipulations on the phenotype, we exposed male Lsamp-deficient mice to environmental enrichment (EE), a technique that has often been shown to abolish phenotypic deviations in knockout mice, and to social isolation, a stressful manipulation, after which all the mice were tested in a behavioral battery. EE abolished differences between the genotypes in body weight and anogenital sniffing, a behavior related to aggressiveness, and amplified the anxiolytic-like phenotype of Lsamp-deficient mice both in the plus-maze and motility box (Innos et al., 2012). Isolation abolished differences between the genotypes in body weight and anxiety and amplified the differences in swimming speed and anogenital sniffing. EE and isolation failed to modify the results as compared to standard housing in whisker trimming, locomotor activity, marble burying, and corticosterone levels. In conclusion, Lsamp-deficient mice were less sensitive to isolation stress than their wild-type littermates. Lack of LAMP protein seemingly leads to a deterioration in the ability to adapt to novel stressful environments and stimuli (Innos et al., 2012).

\section{GENE POLYMORPHISM STUDIES DEMONSTRATING A ROLE OF LSAMP IN MOOD DISORDERS}

Human data link LSAMP not only with anxiety, but also with a wider spectrum of psychiatric disorders: polymorphisms in the human LSAMP gene have been associated with panic disorder (PD; Koido et al., 2006) and male completed suicide (Must et al., 2008). Furthermore, the levels of LAMP protein have been found to be approximately $20 \%$ increased in postmortem frontal cortex both in patients with schizophrenia and bipolar disorder (Behan et al., 2009).

Our recent studies indicate that the LSAMP gene is possibly related to major depressive disorder (MDD) and PD (Koido et al., 2012). We detected statistically significant allelic associations between four LSAMP SNPs and MDD. Two SNPs out of these four contributed to the increased risk (rs1461131 and rs4831089) and two were protective (rs16824691 and rs9874470). Interestingly, all associated SNPs in our study reside in the first intron (1b) and may affect the regulation of gene expression. Only two risk SNPs above were related to PD. Haplotype block was formed from the forenamed SNPs giving three statistically significant haplotypes in the MDD group: TATA was a risk haplotype, whereas CGAG and CGTG were protective haplotypes. The risk haplotype TATA was also statistically significant in the PD group (Koido et al., 2012). On a closer analysis of frequencies of minor alleles of haplotype block, the risk haplotype TATA appeared more frequently than the protective haplotypes CGAG and CGTG in the MDD group ( 41 vs $24 \%$ ) as compared to their respective control group (31 vs $33 \%$; Pearson's Chi square 10.96, $P=0.0009$; Koido et al., 2012). The same trend was observed in the PD group where the risk haplotype TATA was more frequent than the protective haplotypes CGAG and CGTG (38 vs 28\%) as compared to their respective control group ( 31 vs $34 \%$ ). However, the difference in the case of PD did not reach to the level of statistical significance (Pearson's Chi square 3.37, $P=0.066$ ). Therefore, this study (Koido et al., 2012) underlines a significantly stronger relation of the LSAMP gene polymorphisms to MDD than to PD.

\section{STATE OF ART}

In conclusion, the studies described above, performed in laboratory animals and psychiatric patients, suggest a possible role of LAMP in the mechanisms of psychiatric disorders. The Lsamp gene seems to be involved in the regulation of emotional behavior in rodents as the elevated function of the Lsamp gene is accompanied with increased anxiety, whereas the genetic invalidation of the Lsamp gene leads to the reduction of anxiety in rodents. 
There is also a shift in the expression of $\mathrm{GABA}_{\mathrm{A}}$ receptor subunits in favor of alpha2. This could be a reason for the reduced anxiety and increased anxiolytic action of diazepam in Lsampdeficient mice (Innos et al., 2011). Furthermore, the adaptation of Lsamp-deficient mice is impaired in novel and stressful environments and they display reduced aggressiveness. Also, their response to the behavioral effects of amphetamine is blunted (Innos et al., 2013). These behavioral effects are most likely related to changes in the function of the 5-HT-ergic system in Lsamp-deficient mice. Besides that we found a relation between gene polymorphisms of

\section{REFERENCES}

Alttoa, A., Kõiv, K., Hinsley, T. A., Brass, A., and Harro, J. (2010). Differential gene expression in a rat model of depression based on persistent differences in exploratory activity. Eur. Neuropsychopharmacol. 5, 288-300.

Behan, A. T., Byrne, C., Dunn, M. J., Cagney, G., and Cotter, D. R. (2009). Proteomic analysis of membrane microdomain-associated proteins in the dorsolateral prefrontal cortex in schizophrenia and bipolar disorder reveals alterations in LAMP, STXBP1 and BASP1 protein expression. Mol. Psychiatry 14, 601-613.

Catania, E. H., Pimenta, A., and Levitt, P. (2008). Genetic deletion of Lsamp causes exaggerated behavioral activation in novel environments. Behav. Brain Res. 188, 380-390.

Gil, O. D., Zhang, L., Chen, S., Ren, Y. Q., Pimenta, A., Zanazzi, G., et al. (2002). Complementary expression and heterophilic interactions between IgLON family members neurotrimin and LAMP. J. Neurobiol. 51, 190-204.

Horton, H. L., and Levitt, P. (1988). A unique membrane protein is expressed on early developing limbic system axons and cortical targets. J. Neurosci. 12, 4653-4661.

Innos, J., Leidmaa, E., Philips, M.A., Sütt, S., Alttoa, A., Harro, J., et al. (2013). Lsamp ${ }^{-/-}$mice display lower sensitivity to amphetamine and elevated 5-HT turnover. Biochem. Biophys. Res. Commun. 430, 413-418.

Innos, J., Philips, M. A., Leidmaa, E., Heinla, I., Raud, S., Reemann, P., etal. (2011). Lower anxiety and a decrease in agonistic behaviour in Lsamp-deficient mice. Behav. Brain Res. 217, 21-31.

Innos, J., Philips, M.-A., Raud, S., Lilleväli, K., Kõks, S., and Vasar, E.
(2012). Deletion of the Lsamp gene lowers sensitivity to stressful environmental manipulations in mice. Behav. Brain Res. 228, 74-81.

Keller, F., Rimvall, K., Barbe, M. F., and Levitt, P. (1989). A membrane glycoprotein associated with the limbic system mediates the formation of the septo-hippocampal pathway in vitro. Neuron 3, 551-561.

Koido, K., Kõks, S., Must, A., Reimets, A., Maron, E., and Shlik, J. (2006) Association analysis of limbic systemassociated membrane protein gene polymorphisms in mood and anxiety disorders. Eur. Neuropsychopharmacol. 16, S9-S10.

Koido, K., Traks, T., Balõtšev, R., Eller, Associations between LSAMP gene polymorphisms and major depressive disorder and panic disorder. Transl. Psychiatry 2, el52.

Kõks, S., Luuk, H., Nelovkov, A., Areda, T., and Vasar, E. (2004). A screen for genes induced in the amygdaloid area during cat odor exposure. Genes Brain Behav. 3, 80-89.

Kresse, S. H., Ohnstad, H. O., Paulsen, E. B., Bjerkehagen, B., Shuzai, K., Serra, M., etal. (2009). LSAMP, a novel candidate tumor suppressor gene in human osteosarcomas, identified by array comparative genomic hybridization. Genes Chromosomes Cancer 48, 679-693.

Lamprecht, R., Dracheva, S., Assoun, S., and Ledoux, J. E. (2009). Fear conditioning induces distinct patterns of gene expression in lateral amygdala. Genes Brain Behav. 8, 735-743.

Levitt, P. (1984). A monoclonal antibody to limbic system neurons. Science 223, 299-301.

Mann, F., Zhukareva, V., Pimenta, A. Levitt, P., and Bolz, J. (1998). Membrane associated molecules guide T., Must, A., Maron, E., et al. (2012).

the LSAMP gene and MDD (Koido et al., 2012). Patients suffering from MDD have significantly increased ratio between the risk and protective haplotypes of the LSAMP gene compared to healthy subjects. The impact of these haplotypes for the function of LAMP is not clear and remains to be elucidated in future studies.

\section{ACKNOWLEDGMENTS}

This study was supported by grants from the Estonian Science Foundation (8259), the Ministry of Science and Education (SF0180125s08) and the European Regional Development Fund.

limbic and non-limbic thalamocortical projections. J. Neurosci. 18 9409-9419.

Must, A., Tasa, G., Lang, A., Vasar, E., Kõks, S., Maron, E., et al. (2008). Association of limbic system-associated membrane protein (LSAMP) to male completed suicide. BMC Med. Genet. 9:34. doi: 10.1186/1471-2350-9-34

Nelovkov, A., Areda, T., Innos, J., Kõks, S., and Vasar, E. (2006). Rats displaying distinct exploratory activity also have different expression patterns of gamma-aminobutyric acidand cholecystokinin-related genes in brain regions. Brain Res. 1100, 21-31.

Nelovkov, A., Philips, M. A., Kõks, S., and Vasar, E. (2003). Rats with low exploratory activity in the elevated plus-maze have the increased expression of limbic system associated membrane protein gene in the periaqueductal grey. Neurosci. Lett. 352, 179-182.

Pimenta, A. F., Fischer, I., and Levitt, P. (1996). cDNA cloning and structural analysis of the human limbicsystem-associated membrane protein (LAMP). Gene 170, 189-195.

Pimenta, A. F., Zhukareva, V., Barbe, M. F., Reinoso, B. S., Grimley, C., Henzel, W., et al. (1995). The limbic systemassociated membrane protein is an Ig superfamily member that mediates selective neuronal growth and axon targeting. Neuron 15, 287-297.

Qiu, S., Champagne, D. L., Peters, M. Catania, E. H., Weeber, E. J., Levitt, P., et al. (2010). Loss of limbic systemassociated membrane protein leads to reduced hippocampal mineralocorticoid receptor expression, impaired synaptic plasticity, and spatial memory deficit. Biol. Psychiatry 68, 197-204.

Reinoso, B. S., Pimenta, A. F., and Levitt, P. (1996). Expression of the mRNAs encoding the limbic system-associated membrane protein (LAMP): II. Adult rat brain. J. Comp. Neurol. 375, 274-288.

Schalkwyk, L. C., Fernandes, C., Nash, M. W., Kurrikoff, K., Vasar, E., and Kõks, S. (2007). Interpretation of knockout experiments: the congenic footprint. Genes Brain Behav. 3, 299-303.

Zacco, A., Cooper, V., Chantler, P. D., Fisher-Hyland, S., Horton, H. L., and Levitt, P. (1990). Isolation, biochemical characterization and ultrastructural analysis of the limbic system associated membrane protein (LAMP), a protein expressed by neurons comprising functional neural circuits. J. Neurosci. 10, 73-90.

Conflict of Interest Statement: The authors declare that the research was conducted in the absence of any commercial or financial relationships that could be construed as a potential conflict of interest.

Received: 23 December 2012; accepted: 08 March 2013; published online: 26 March 2013.

Citation: Innos J, Koido K, Philips M$A$ and Vasar E (2013) Limbic systemassociated membrane protein as a potential target for neuropsychiatric disorders. Front. Pharmacol. 4:32. doi: 10.3389/ fphar.2013.00032

This article was submitted to Frontiers in Neuropharmacology, a specialty of Frontiers in Pharmacology.

Copyright (c) 2013 Innos, Koido, Philips and Vasar. This is an open-access article distributed under the terms of the Creative Commons Attribution License, which permits use, distribution and reproduction in other forums, provided the original authors and source are credited and subject to any copyright notices concerning any third-party graphics etc. 